\title{
ON THE FINITENESS OBSTRUCTION OF CERTAIN PERIODIC GROUPS
}

\author{
STEFFEN BENTZEN
}

\section{Introduction.}

For groups $G$ with periodic cohomology, the Tate cohomology groups $\hat{H}^{n d}(G, Z)$ are isomorphic to $\mathrm{Z} /|G|$ for all multiples of the period $d$. Each generator $e$ of $\hat{H}^{\text {nd }}(G, Z)$ can be realized by a periodic resolution $P_{*}$ of $Z$ by finitely generated projective $Z G$-modules, and the resolution can be taken to be free exactly if the element

$$
\sigma_{n d}(e):=\sum_{i=1}^{n d-1}(-1)^{i}\left[P_{i}\right]
$$

vanishes in $\tilde{K}_{0}(\mathrm{ZG})=\mathrm{Cl}(G)$. This element $\sigma_{n d}(e)$ is called the finiteness obstruction because its vanishing is equivalent to the existence of a free simplicial action of $G$ on a finite simplicial complex $\Sigma$, homotopy equivalent to the (nd-1)-dimensional sphere, such that $\Sigma / G$ has $e$ as its first $k$-invariant. The calculation of $\sigma_{n d}$ is therefore of interest from a topological as well as from an algebraic point of view.

By results of [8] the groups of particular topological importance are the groups $Q(8 a, b, c) \times Z / d$. In this paper we consider the group $Q(8 a, b)$ which is a semi-direct product of $\mathrm{Z} / a b$ with $Q(8)$ such that the kernel of the action of $Q(8)$ on $\mathrm{Z} / a b$ is $\mathrm{Z} / 2$. This group has cohomology of period 4. By Corollary $\mathrm{C}$ of [4] there is a special class of generators of $\bar{H}^{4}(Q(8 a, b), \mathrm{Z})$ that is of interest for the topological space form problem; these are the almost linear generators considered below. We take such an almost linear generator $e_{0}$ and calculate $\sigma_{4}\left(e_{0}\right)$ (Theorem 1). The result is a generalization of Milgram's result for $Q(8 p, q)$ where $p$ and $q$ are primes, cf. [5] or [1].

Unfortunately, Theorem 1 gives the answer in terms of non-trivial number theoretical questions. If both $a$ and $b$ are prime powers, these questions have been almost completely settled in [1].

Before stating the main result, we fix some notation that will remain in force throughout the paper.

Like in [1] we use Fröhlich's description of $\mathrm{Cl}(G)$ in terms of character

Received April 19, 1984. 
homomorphisms. For details on this formalism we refer to [2] and the appendix of [3], but let us briefly sketch the basic features. Let $H$ be a Galois functor from the category of extension fields of $Q$ to abelian groups. It can be extended to a functor on commutative finite-dimensional semi-simple algebras over Q by letting $H\left(\prod L_{i}\right)=\Pi H\left(L_{i}\right)$. Then there is a basic isomorphism

$$
H(Z(\mathrm{Q} G)) \cong \operatorname{Hom}_{\Omega}(R(G), H(\bar{Q}))
$$

for any finite group $G$. Here $Z$ denotes the center, $\Omega$ is the full Galois group of $\mathrm{Q}$, and $R(G)$ is the virtual character ring of $G$. The left hand side is convenient for notational purposes while the right hand side is convenient for calculations, and we shall constantly change from one side to the other.

Taking the exact sequence

$$
Z(\mathrm{Q} G)_{+}^{\times} \rightarrow J(Z(\mathrm{Q} G))_{+} / \mathrm{Nrd}(U(\mathrm{Z} G)) \stackrel{\partial}{\rightarrow} \mathrm{Cl}(G) \rightarrow 0
$$

of [2, Theorem 2] and using (0.1) we get

$$
\operatorname{Hom}_{\Omega}^{+}\left(R(G), \overline{\mathrm{Q}}^{\times}\right) \rightarrow \operatorname{Hom}_{\Omega}^{+}(R(G), J(\overline{\mathrm{Q}})) / \operatorname{Det}(U(\mathrm{Z} G)) \stackrel{\partial}{\rightarrow} \mathrm{Cl}(G) \rightarrow 0,
$$

where $U(Z G)$ is the group of unit ideles:

$$
U(Z G)=\prod_{p}\left(Z_{p} G\right)^{\times}
$$

with $p$ running through all primes, including the infinite, cf. [3, 1.10]. Similarly there is an exact sequence

$$
\begin{aligned}
\operatorname{Hom}_{\Omega}^{+}\left(R(G), \mathcal{O}_{F}^{\times}\right) & \left.\rightarrow \operatorname{Hom}_{\Omega}\left(R(G), \prod_{p \| G \mid} \mathcal{O}_{F_{p}}^{\times}\right) / \operatorname{Det}\left(\prod_{p \| G \mid} Z_{p} G\right) \times\right) \\
& \rightarrow D(G) \rightarrow 0
\end{aligned}
$$

describing the kernel group, [F2, II.2]. Here $F$ is any number field containing all character values, and $\operatorname{Hom}_{\Omega}^{+}\left(R(G), \mathcal{O}_{F}^{+}\right)$denotes the character homomorphisms $f$ for which $f(\chi)$ is real and positive at every infinite prime (real or complex) of $F$ whenever $\chi$ is symplectic. The middle term in (0.4) decomposes into

$$
\prod_{p|| G \mid} \operatorname{Hom}_{\Omega}\left(R(G), \mathcal{O}_{F}^{\times}\right) / \operatorname{Det}\left(\left(Z_{p} G\right)^{\times}\right) .
$$

The component at $p$ is called the local kernel group (at $p$ ) and is denoted $\hat{D}_{p}(G)$. The whole product is denoted $\hat{D}(G)$.

A basic feature of the character homomorphism formalism is that the restriction and induction maps on class groups are determined through induction and restriction of characters, cf. [3, VI and VIII]. 
We are considering the group $\pi=Q(8 a, b)$ which is defined as follows. Let

$$
Q(8)=\left\langle X, Y \mid X^{4}=Y^{4}=1, X^{2}=Y^{2} \neq 1, X Y X^{-1}=Y^{-1}\right\rangle
$$

be the quaternion group of order 8 . Let $X$ act on $Z / a \times Z / b$ by inversion on $Z / a$ and the identity on $\mathrm{Z} / b$, and let $Y$ act by inversion on $\mathrm{Z} / b$ and the identity on $\mathrm{Z} / a$. This gives an action on $Q(8)$ on $\mathrm{Z} / a b$ and $Q(8 a, b)$ is the semi-direct product $\mathrm{Z} / a b \times Q(8)$ defined through this action.

If $a=\prod_{i} p_{i}^{n_{1}}, b=\prod_{i} q_{i}^{m_{1}}$ is the prime decomposition of $a$ and $b$ we let $P_{1}$ $=\left\{p_{i}^{n_{1}} \mid i\right\}, P_{2}=\left\{q_{i}^{m_{1}} \mid i\right\}$, and $P=P_{1} \cup P_{2}$. With an abuse of language we shall by a statement like " $p \in P_{1}$ " mean that for some power $p^{n}$ of $p$ we have $p^{n} \in P_{1}$. Likewise, by $P_{1} \backslash\{p\}$ we shall mean $P_{1} \backslash\left\{p^{n}\right\}$. For each set $S \leqq P$, let $\pi(S)$ be the subgroup of $\pi$ obtained by deleting the prime powers not in $S$. Let further $C=C_{\pi}(\mathrm{Z} / a b)$ be the cyclic subgroup of $\pi$ of order $2 a b$, centralizing $\mathrm{Z} / a b$.

For each prime power $p^{n}$ in $P$ we choose a generator $A_{p}$ of $Z / p^{n}$, and for each $A_{p}$ we let $X_{p}$ and $Y_{p}$ be defined by: If $X^{-1} A_{p} X=A_{p}$, we let $X_{p}=X$ and $Y_{p}=Y$. If $Y^{-1} A_{p} Y=A_{p}$ we let $X_{p}=Y$ and $Y_{p}=X$. Furthermore we let for each $A_{p}, \chi_{p}$ be the generator of $\mathrm{Ch}\left(\mathrm{Z} / p^{n}\right)$ with $\chi_{p}\left(A_{p}\right)=e^{2 \pi i / p^{n}}$, and we let $\chi_{2}$ be the unique irreducible representation of $Q(8)$ of dimension 2.

As mentioned, $\pi$ has cohomology of period 4 . For each $p^{n} \in P$, let

$$
e_{p}=c_{2}\left(\chi_{p}+\chi_{p}^{-1}\right) \in \hat{H}^{4}\left(Z / p^{n}, Z\right)
$$

and let

$$
e_{2}=c_{2}\left(\chi_{2}\right) \in \hat{H}^{4}(Q(8), Z) .
$$

Here $c_{2}$ denotes the second Chern class. These $e$ 's are generators of the different cohomology groups, and we define $e_{0}$ to be the generator of $\hat{H}^{4}(Q(8 a, b), \mathrm{Z})$ that restricts to these $e$ 's under the isomorphism

$$
\hat{H}^{4}(Q(8 a, b), \mathrm{Z}) \cong \prod_{p^{n} \in P} \hat{H}^{4}\left(\mathrm{Z} / p^{n}, \mathrm{Z}\right) \oplus \hat{H}^{4}(Q(8), \mathrm{Z}) .
$$

This is the almost linear generator for which we shall calculate $\sigma_{4}\left(e_{0}\right)$.

Once and for all we make a choice of roots of unity: If $n=p^{m}$, then $\zeta_{n}=e^{2 \pi i / p^{m}}$, and if $n=\prod p_{i}^{n_{1}}$, then $\dot{\zeta}_{n}=\prod \zeta_{p_{i}^{n_{*}}}$ Further, for any $n \in \mathrm{N}$ we let $\eta_{n}=\zeta_{n}+\zeta_{n}^{-1}$.

For any number ring $A$ we let $A^{\times}$denote the units of $A$, and, if $A$ is real, we let $A^{*}$ denote the totally positive units. For any finite prime $\not$ of $A$ we let $\varrho_{/}$ denote the reduction map $\varrho_{h}: A \rightarrow A / h$.

Our main result is

THEOREM $1 . \sigma_{4}\left(e_{0}\right)$ is of order at most 2 ; it lies in the kernel group $D(\pi)$ and it is zero if and only if for any pair $(\alpha, \beta)$ of square free numbers with $\alpha|\beta, \beta| b$ and $\alpha, \beta$ $\neq 1$ there exist numbers $x_{\alpha, \beta} \in \mathbb{Z}\left[\eta_{\alpha}, \eta_{\beta}\right]^{\times}, y_{\alpha, \beta} \in \mathbb{Z}\left[\eta_{\alpha}, \eta_{\beta}\right]^{*}$ such that 


$$
\begin{aligned}
& \varrho_{h}\left(x_{\alpha, \beta}\right)=\left\{\begin{aligned}
1 & \text { if } \not \mid \alpha \\
-1 & \text { if } \not \mid \beta
\end{aligned}\right. \\
& \varrho_{h}\left(y_{x, \beta}\right)=4 \text { for } \not \mid \alpha \beta
\end{aligned}
$$

Note that since $\sigma_{4}\left(e_{0}\right)$ is 2-torsion, we can in Theorem 1 replace 4 by its 2primary part in $\left(Z\left[\eta_{\alpha}, \eta_{\beta}\right] / h\right)^{\times}$.

\section{Decomposition of the class group.}

For $p \in P$, let $E_{p}$ be the idempotent endomorphism of $\pi$ that sends the Sylow-p part to 1 and is the identity on the rest. It factors as

$$
E_{p}: \pi \rightarrow \pi(P \backslash\{p\}) \rightarrow \pi,
$$

where $\pi \rightarrow \pi(P \backslash\{p\})$ is the projection and $\pi(P \backslash\{p\}) \rightarrow \pi$ is the inclusion. If no power of $p$ is in $P$, we let $E_{p}$ be the identity. These maps are defined on all the subgroups $\pi(S)$ and $C$ of $\pi$, and they commute with the inclusion maps. Furthermore these endomorphisms will induce commuting idempotents on the virtual character rings $R(\pi(S))$ and $R(C)$ and on the projective class groups $\mathrm{Cl}(\pi(S)), \mathrm{Cl}(C)$.

Lemma 1.1. On the class groups we have
a) $\operatorname{Res}_{\pi\left(P \backslash\left\{p_{i}\right)^{\circ}\right.}^{\pi} E_{p}=\operatorname{Res}_{\pi(P \backslash\{p\})}^{\pi}$
for all $p \in P$
b) $\operatorname{Res}_{\pi(P \backslash\{q\})}^{\pi} \circ E_{p}=E_{p} \circ \operatorname{Res}_{\pi(P \backslash\{q\})}^{\pi} \quad$ for all $q \in P \backslash\{p\}$
c) $\operatorname{Res}_{C}^{\pi} E_{p} \quad=E_{p} \circ \operatorname{Res}_{C}^{\pi} \quad$ for all $p \in P$.

\section{Proof. Obvious.}

Note. Concerning (c) of Lemma 1.1 we actually have a stronger result: $E_{p}$ commutes with induction of characters from $C$ to $\pi$, so the diagram

$$
\begin{array}{rr}
R(C) \stackrel{\text { Ind }}{\longrightarrow} & R(\pi) \\
\downarrow E_{p} & \downarrow E_{p} \\
R(C) \stackrel{\text { Ind }}{\longrightarrow} & R(\pi)
\end{array}
$$

is commutative.

For any $S \cong P$, let

$$
\tilde{E}_{S}=\prod_{p \notin S} E_{p} \prod_{p \in S}\left(1-E_{p}\right) .
$$

Then, on the character ring $R(\pi)$ : 


$$
1=\sum_{S \cong P} \tilde{E}_{S}
$$

is a decomposition of the identity into a sum of orthogonal idempotents, i.e. projections. This gives a decomposition

$$
\mathrm{Cl}(\pi)=\prod_{S \cong P} \tilde{E}_{S}(\mathrm{Cl}(\pi))
$$

of the class groups.

According to Lemma 1.1 we have

$$
\operatorname{Res}_{\pi(S)}^{\pi} \circ \tilde{E}_{S}=\tilde{E}_{S} \circ \operatorname{Res}_{\pi(S)}^{\pi}
$$

on the class groups. Hence we get

Corollary 1.2. The restriction to $\pi(S)$ of the $S$-component is the top component of the restriction.

By the top component of $\mathrm{Cl}(\pi)$ is meant $\widetilde{E}_{P}(\mathrm{Cl}(\pi))$.

\section{Detecting the components.}

We begin by detecting the 2-primary part of the non top components.

Proposition 2.1. If $S \Phi P$, then

$$
\operatorname{Res}_{\pi(S)}^{\pi}: \tilde{E}_{S}(\mathrm{Cl}(\pi)) \rightarrow \mathrm{Cl}(\pi(S))
$$

is injective on the 2-primary part.

Proof. In Fröhlich's description of the class group

$$
\mathrm{Cl}(\pi)=\operatorname{Hom}_{\Omega}(R(\pi), J(\overline{\mathrm{Q}})) / \operatorname{Det}\left(U(\mathrm{Z}(\pi)) \operatorname{Hom}_{\Omega}\left(R(\pi), \overline{\mathrm{Q}}^{\times}\right),\right.
$$

and $\operatorname{Res}_{\pi(S)^{\circ}}^{\circ} \prod_{p \notin S} E_{p}$ is induced by the map

$$
\begin{aligned}
f: R(\pi(S)) \stackrel{\text { Ind }}{\longrightarrow} R(\pi) \stackrel{\text { Res }}{\longrightarrow} R\left(\pi\left(P \backslash\left\{p_{1}\right\}\right)\right) \stackrel{\text { lift }}{\longrightarrow} R(\pi) \stackrel{\text { Res }}{\longrightarrow} \\
R\left(\pi\left(P \backslash\left\{p_{2}\right\}\right)\right) \stackrel{\text { lift }}{\longrightarrow} R(\pi) \rightarrow \text { etc. }
\end{aligned}
$$

where $P \backslash S=\left\{p_{1}, p_{2}, \ldots\right\}$. Obviously the diagram

$$
R\left(\pi\left(P \backslash\left\{p_{i}\right\}\right)\right) \stackrel{\text { lift }}{\longrightarrow} R(\pi) \stackrel{\text { Res }}{\longrightarrow} R\left(\pi\left(P \backslash\left\{p_{j}\right\}\right)\right)
$$

is commutative. Hence $f$ is the composite map: 


$$
R(\pi(S)) \stackrel{\text { Ind }}{\longrightarrow} R(\pi) \stackrel{\text { Res }}{\longrightarrow} R(\pi(S)) \stackrel{\text { lift }}{\longrightarrow} R(\pi) .
$$

To prove the proposition it suffices to prove that ResoInd: $R(\pi(S)) \rightarrow R(\pi(S))$ induces a map on the class group $\mathrm{Cl}(\pi(S))$ that is injective on the 2-primary part. We prove this by proving that this induced map is of the form id $+g$, where $g \circ g=2 \cdot g_{0}$ for some map $g_{0}$. If $x$ is 2-primary and $x+g(x)=0$, then $x=2^{i} \cdot g_{0}^{i}(x)$ for all $i>0$, and hence $x=0$.

We apply the Mackey decomposition to Res $\circ$ Ind. We have the double cosets

$$
\begin{aligned}
\pi=\pi(S) \cup & \pi(S) A_{p_{1}} \pi(S) \cup \ldots \cup \pi(S) A_{p_{1}}^{\left(p_{1}^{n_{1}}-1\right) / 2} \pi(S) \\
& \cup \pi(S) A_{p_{2}} \pi(S) \cup \ldots \cup \pi(S) A_{p_{2}}^{\left(p_{2}^{n_{2}}-1\right) / 2} \pi(S) \\
& \cup \text { etc. }
\end{aligned}
$$

and

$$
A_{p}^{j} \pi(S) A_{p}^{-j} \cap \pi(S)=\left\langle A_{l}, X_{p} \mid l \in S\right\rangle=\tau(S, p) .
$$

Therefore

$$
(\operatorname{Res} \circ \operatorname{Ind})(\chi)=\chi+\sum_{p \notin S}\left(\left(p^{n}-1\right) / 2\right) \cdot N_{p}(\chi),
$$

where $N_{p}$ is the map

$$
N_{p}=\operatorname{Ind}_{\tau(S, p)}^{\pi(S)} \circ \operatorname{Res}_{\tau(S, p)}^{\pi(S)} .
$$

If $p \in P_{1}, q \in P_{2}$, the Mackey decomposition gives

$$
\begin{aligned}
N_{p} \circ N_{q} & =\operatorname{Ind}_{\tau(S, p)}^{\pi(S)} \circ \operatorname{Res}_{\tau(S, p)}^{\pi(S)} \circ \operatorname{Ind}_{\tau(S, q)}^{\pi(S)} \circ \operatorname{Res}_{\tau(S, q)}^{\pi(S)} \\
& =\operatorname{Ind}_{\tau(S, p)}^{\pi(S)} \circ \operatorname{Ind}_{\gamma}^{\tau(S, p)} \circ \operatorname{Res}_{\gamma}^{\tau(S, q)} \circ \operatorname{Res}_{\tau(S, q)}^{\pi(S)} \\
& =\operatorname{Ind}_{\gamma}^{\pi(S)} \operatorname{Res}_{\gamma}^{\pi(S)},
\end{aligned}
$$

where $\gamma=\left\langle A_{l}, X_{p}^{2} \mid l \in S\right\rangle$.

If $p, q \in P_{1}$ or $p, q \in P_{2}$, the Mackey decomposition gives

$$
N_{p} \circ N_{q}=\operatorname{Ind}_{\tau(S, p)}^{\pi(S)} \circ\left(\operatorname{id}+\mathscr{C}\left(Y_{p}\right)\right) \circ \operatorname{Res}_{\tau(S, p)}^{\pi(S)},
$$

where $\mathscr{C}\left(Y_{p}\right)$ is conjugation by $Y_{p}$. Hence, the $N_{p}$ 's are commuting operators satisfying $N_{p} \circ N_{p}=2 \cdot N_{p}$. Putting

$$
N=\sum_{p \notin S}\left(\left(p^{n}-1\right) / 2\right) \cdot N_{p},
$$

there exists an operator $N_{0}$ such that $N \circ N=2 \cdot N_{0}$. This completes the proof of the Proposition.

Now we turn to the top component. We shall not detect the top component 
of the class group. Instead we detect the top component of the middle term in the exact sequence $(0.2)$.

We shall prove

Proposition 2.2. $\operatorname{Res}_{C}$ is injective on

$$
\left[\tilde{E}_{p}\left(J(Z(\mathrm{Q} \pi))_{+} / \mathrm{Nrd}(U(\mathrm{Z} \pi))\right)\right]_{(2, f)},
$$

where the subscript $(2, f)$ denotes the 2-primary part plus the free part.

To prove Proposition 2.2 we shall actually have to calculate the 2-primary parts of the local kernel groups $\hat{D}_{p}(\pi)$. To state the result, we need some terminology. For $p$ a prime and $x$ an integer, we let $x_{p}^{\prime}$ denote the $p$-free part of $x$. For two pairs $(\alpha, \beta)$ and $(x, y)$ of integers we call $(\alpha, \beta)$ full with respect to $(x, y)$ if $\alpha|\mathrm{x}, \beta| \mathrm{y}$ and any prime that divides $x$ (respectively $y$ ) also divides $\alpha$ (respectively $\beta$ ). Then

Proposition 2.3. The top component of $\hat{D}_{p}(\pi)_{(2)}(p \mid a)$ splits into a direct sum $D_{p,+} \oplus D_{p,-}$, where both $D_{p,+}$ and $D_{p,-}$ are isomorphic to

$$
\prod_{(\alpha, \beta)} \prod_{i=1}^{n(p)}\left(\boldsymbol{F}_{p} \otimes \mathbb{Z}\left[\eta_{\alpha}, \eta_{\beta}\right]\right)_{(2)}^{\times} .
$$

Here $(\alpha, \beta)$ runs through the pairs of (positive) integers that are full with respect to $\left(a_{p}^{\prime}, b\right)$ and $p^{n(p)}$ is the exact power of $p$ dividing a. Furthermore, the map

$$
\tilde{E}_{P}\left(\Phi_{p}\right): \tilde{E}_{P}\left(\mathcal{O}_{\mathrm{Z}}^{\times}(\mathrm{Q} \pi),+\right) \rightarrow \tilde{E}\left(\hat{D}_{p}(\pi)\right)_{(2)}
$$

in the exact sequence describing $\widetilde{E}_{P}(D(\pi))_{(2)}$ factors into $\Phi_{p,+} \oplus \Phi_{p,-}$, where

$$
\Phi_{p,+}: \prod_{(\alpha, \beta)} \prod_{i=1}^{n(p)} \mathrm{Z}\left[\eta_{\alpha p^{i}}, \eta_{\beta}\right]^{\times} \rightarrow \prod_{(\alpha, \beta)}\left(F_{p} \otimes Z\left[\eta_{\alpha}, \eta_{\beta}\right]\right)_{(2)}^{\times}
$$

and

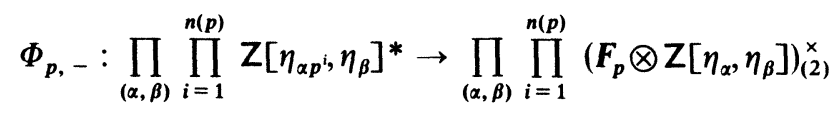

are the natural reduction maps. Furthermore, the top component of $\hat{D}_{2}(\pi)_{(2)}$ is trivial.

Proof (of Propositions 2.2 and 2.3). First of all we have the exact sequence

$$
0 \rightarrow U(Z(\mathrm{Q} \pi))_{+} / \mathrm{Nrd}(U(\mathrm{Z} \pi)) \rightarrow J(Z(\mathrm{Q} \pi))_{+} / \mathrm{Nrd}(U(\mathrm{Z} \pi)) \rightarrow I(\pi) \rightarrow 0 .
$$

Here $U(Z(\mathrm{Q} \pi))$ is the group of unit ideles of $Z(\mathrm{Q} \pi)$, and $I(\pi)$ is nothing but the 
product of the ideal groups of the field components of $Z(\mathrm{Q} \pi)$. Since $I(\pi)$ is free and $U(Z(\mathrm{Q} \pi))_{+} / N r d(U(Z \pi))$ is finite, we get the split exact sequence

$$
\begin{aligned}
0 \rightarrow\left[U(Z(\mathrm{Q} \pi))_{+} / N r d(U(\mathrm{Z} \pi))\right]_{(2)} & \rightarrow\left[J(Z(\mathrm{Q} \pi))_{+} / N r d(U(\mathrm{Z} \pi))\right]_{(2, f)} \rightarrow \\
& \rightarrow I(\pi) \rightarrow 0 .
\end{aligned}
$$

Here,

$$
\left.U(Z(\mathrm{Q} \pi))_{+} / \operatorname{Nrd}(U(\mathrm{Z} \pi))=\prod_{l|| \pi \mid}\left[\left(\mathcal{O}_{Z(\mathrm{Q} \pi)}\right)^{\times} / \operatorname{Nrd}\left(\mathrm{Z}_{l} \pi\right)^{\times}\right)\right]=\hat{D}(\pi)
$$

since $Z_{l} \pi$ is a maximal order (respectively $Q_{l} \pi$ ) for $l \nmid|\pi|$ (respectively $l$ infinite). We get the diagram

$$
\begin{gathered}
0 \rightarrow \hat{D}(\pi) \rightarrow J(Z(\mathrm{Q} \pi))_{+} / \operatorname{Nrd}(U(\mathrm{Z} \pi)) \rightarrow I(\pi) \rightarrow 0 \\
\downarrow \operatorname{Res} \\
0 \rightarrow \hat{D}(C) \longrightarrow J(\mathrm{Q} C) / \operatorname{Nrd}(U(\mathrm{Z} C)) \longrightarrow I(C) \rightarrow 0
\end{gathered}
$$

and Proposition 2.2 will be proved if we prove that Res is injective on $\left(\tilde{E}_{P} \hat{D}(\pi)\right)_{(2)}$ and on $\tilde{E}_{P} I(\pi)$.

The group algebra decomposes as

$$
\begin{aligned}
\mathrm{Q} \pi= & \mathrm{Q}_{++} \oplus \mathrm{Q}_{+-} \oplus \mathrm{Q}_{-+} \oplus \mathrm{Q}_{--} \oplus \mathrm{H}_{\mathrm{Q}} \\
& \oplus \prod_{\substack{\alpha \mid a \\
\alpha \neq 1}}\left(M_{2}\left(\mathrm{Q}\left(\eta_{\alpha}\right)\right)_{Y=1} \oplus M_{2}\left(\mathrm{Q}\left(\eta_{\alpha}\right)\right)_{Y=-1} \oplus \mathrm{H}_{\mathrm{Q}\left(\eta_{4 \alpha}\right)}\right) \\
& \oplus \prod_{\substack{\beta \mid b \\
\beta \neq 1}}\left(M_{2}\left(\mathrm{Q}\left(\eta_{\beta}\right)\right)_{X=1} \oplus M_{2}\left(\mathrm{Q}\left(\eta_{\beta}\right)\right)_{X=-1} \oplus \mathrm{H}_{\mathrm{Q}\left(\eta_{\alpha \beta}\right)}\right) \\
& \oplus \prod_{\substack{\alpha|a, \beta| b \\
\alpha, \beta \neq 1}}\left(M_{4}\left(\mathrm{Q}\left(\eta_{\alpha}, \eta_{\beta}\right)\right) \oplus A\left(\mathrm{Q}\left(\zeta_{\alpha \beta}\right) / \mathrm{Q}\left(\eta_{\alpha}, \eta_{\beta}\right)\right)\right) .
\end{aligned}
$$

Here $A\left(\mathrm{Q}\left(\zeta_{\alpha \beta}\right) / \mathrm{Q}\left(\eta_{\alpha}, \eta_{\beta}\right)\right)$ is a central simple algebra over $\mathrm{Q}\left(\eta_{\alpha}, \eta_{\beta}\right)$. This we shall not specify further; we only note that it does not split at $R$. Furthermore, $\mathrm{Q}_{++} \oplus \mathrm{Q}_{+-} \oplus \mathrm{Q}_{-+} \oplus \mathrm{Q}_{--} \oplus \mathrm{H}_{\mathbf{Q}}$ is the decomposition of $\mathrm{Q} Q(8)$, where $\mathrm{Q}_{++}$ (say) is the summand corresponding to $X=1, Y=1$.

We choose representatives for $\operatorname{Irr}(\pi) / \Omega$ corresponding to this decomposition, and we choose them in accordance with our choice of roots of unity (cf. section 0). Let us denote these characters

$$
\begin{array}{lll}
x_{1}, & x_{2}, & x_{3}, x_{4}, x_{5} \\
x_{\alpha, 1}, & x_{\alpha,-1}, & x_{\alpha, 0} \\
y_{\beta, 1}, & y_{\beta,-1}, & y_{\beta, 0} \\
z_{\alpha, \beta, 1}, & z_{\alpha, \beta,-1} .
\end{array}
$$


Then we can tabulate the action of the idempotent $E_{p}$ on $R(\pi)$ as in Table 2.4, where $\sigma$ is the generator of $\operatorname{Gal}(\mathrm{Q}(i) / \mathrm{Q})$.

\begin{tabular}{|c|c|c|c|}
\hline & $\chi$ & & $E_{p}(\chi)$ \\
\hline$x_{i}$ & $i=1, \ldots, 5$ & & $x_{i}$ \\
\hline$x_{\alpha, 1}$ & & $x_{\alpha_{p}^{\prime} 1}$ & (or $x_{1}+x_{3}$ if $\alpha$ is a power of $p$ ) \\
\hline$x_{\alpha,-1}$ & & $x_{x_{p}^{\prime},-1}$ & (or $x_{2}+x_{4}$ if $\alpha$ is a power of $p$ ) \\
\hline$x_{\alpha, 0}$ & & $x_{x_{p}^{\prime}, 0}$ & (or $x_{5}$ if $\alpha$ is a power of $p$ ) \\
\hline $\begin{array}{l}y_{\beta, i} \\
z_{\alpha, \beta, 1} \\
z_{\alpha, \beta-1}\end{array}$ & $i=-1,0,1$ & $\begin{array}{l}z_{\alpha_{p}^{\prime}, \beta, 1} \\
z_{\alpha_{p}^{\prime}, \beta,-1}\end{array}$ & $\begin{array}{l}y_{\beta, i} \\
\text { (or } y_{\beta, 1}+y_{\beta,-1} \text { if } \alpha \text { is a power of } p \text { ) } \\
\text { (or } y_{\beta, 0}+y_{\beta, 0}^{\sigma} \text { if } \alpha \text { is a power of } p \text { ) }\end{array}$ \\
\hline
\end{tabular}

Table 2.4

From this table we see that $\tilde{E}_{P}=\prod_{l \in P}\left(1-E_{l}\right)$ maps all characters to zero, except those $z_{\alpha, \beta, 1}$ and $z_{\alpha, \beta,-1}$, where $(\alpha, \beta)$ is full with respect to $(a, b)$. Hence the top component of $R(\pi) / \Omega$ is equal to

$$
\prod_{\substack{(,, \beta) \\ \text { full }}} \mathrm{Z} \cdot z_{\alpha, \beta, 1} \oplus \prod_{\substack{(\alpha, \beta) \\ \text { full }}} \mathrm{Z} \cdot z_{\alpha, \beta,-1} .
$$

To calculate $\operatorname{Res}_{C}^{\pi}$ on $\operatorname{Hom}_{\Omega}(R(\pi), J(\bar{Q}))$ we must induce characters from $C$ to $\pi$. We decompose

$$
\mathrm{Q} C=\prod_{\left.\substack{\alpha \\ \beta}\right|_{b} ^{a}}\left(\mathrm{Q}\left(\zeta_{\alpha \beta}\right)_{T=1} \oplus \mathrm{Q}\left(\zeta_{\alpha \beta}\right)_{T=-1}\right),
$$

where $T$ is the involution of $C$. We choose representatives of $\operatorname{Irr}(C) / \Omega$ according to our choice of roots of unity, and we denote these

$$
w_{\alpha, \beta, 1}, w_{\alpha, \beta,-1} \quad \alpha|a, \beta| b .
$$

These characters will induce as tabulated in Table 2.5 .

\begin{tabular}{ll|l}
\multicolumn{1}{c|}{$\chi$} & \multicolumn{1}{c}{$\operatorname{Ind}_{C}(\chi)$} \\
\hline$w_{1,1,1}$ & & $x_{1}+x_{2}+x_{3}+x_{4}$ \\
$w_{1,1,-1}$ & & $2 \cdot x_{5}$ \\
$w_{\alpha, 1,1}$ & $\alpha \neq 1$ & $x_{\alpha, 1}+x_{\alpha,-1}$ \\
$w_{\alpha, 1,-1}$ & $\alpha \neq 1$ & $x_{\alpha, 0}+x_{\alpha, 0}^{\sigma}$ \\
$w_{1, \beta, 1}$ & $\beta \neq 1$ & $y_{\beta, 1}+y_{\beta,-1}$ \\
$w_{1, \beta,-1}$ & $\beta \neq 1$ & $y_{\beta, 0}+y_{\beta, 0}^{\sigma}$ \\
$w_{\alpha, \beta, 1}$ & $\alpha, \beta \neq 1$ & $z_{\alpha, \beta, 1}$ \\
$w_{\alpha, \beta,-1}$ & $\alpha, \beta \neq 1$ & $z_{\alpha, \beta,-1}$ \\
\hline
\end{tabular}

Table 2.5. 
It follows that on $\tilde{E}_{P}(J(Z(\mathrm{Q} \pi)))$, Res is the inclusion

$$
\prod_{\substack{(\alpha, \beta) \\ \text { full }}}\left(J\left(\mathrm{Q}\left(\eta_{\alpha}, \eta_{\beta}\right)\right) \oplus J\left(\mathrm{Q}\left(\eta_{\alpha}, \eta_{\beta}\right)\right)\right) \rightarrow \prod_{\substack{(\alpha, \beta) \\ \text { full }}}\left(J\left(\mathrm{Q}\left(\zeta_{\alpha \beta}\right)\right) \oplus J\left(\mathrm{Q}\left(\zeta_{\alpha \beta}\right)\right)\right)
$$

and on $\tilde{E}_{P}(I(\pi))$, Res is the inclusion

$$
\prod_{\substack{(\alpha, \beta) \\ \text { full }}}\left(I\left(\mathrm{Q}\left(\eta_{\alpha}, \eta_{\beta}\right)\right) \oplus I\left(\mathrm{Q}\left(\eta_{\alpha}, \eta_{\beta}\right)\right)\right) \rightarrow \prod_{\substack{(\alpha, \beta) \\ \text { full }}}\left(I\left(\mathrm{Q}\left(\zeta_{\alpha \beta}\right)\right) \oplus I\left(\mathrm{Q}\left(\zeta_{\alpha \beta}\right)\right)\right),
$$

Now we turn to $\tilde{E}_{P}(\hat{D}(\pi))$. Let $p|| \pi \mid, p \neq 2$. We shall calculate $\tilde{E}_{P}\left(\hat{D}_{p}(\pi)\right)_{(2)}$, so we decompose $Z_{p} \pi$ and calculate the $p^{\prime}$-part of $\tilde{E}_{P}\left(\hat{D}_{p}(\pi)\right)$. Let us assume $p \mid a$.

$$
\begin{aligned}
Z_{p} \pi & =2 \cdot Z_{p}\left[Z / p^{n}\right]^{t}\left[X \mid X^{2}=1\right]_{Y= \pm 1} \\
& \oplus 2 \cdot \prod_{\substack{\alpha \mid \alpha_{p}^{\prime} \\
\alpha \neq 1}}\left(\left(Z_{p} \otimes Z\left[\zeta_{\alpha}\right]\right)\left[Z / p^{n}\right]\right)^{t}\left[X \mid X^{2}=1\right] \\
& \oplus \prod_{\substack{\alpha\left|a_{p}^{\prime} \beta\right| b \\
\beta \neq 1}}\left(\left(Z_{p} \otimes Z\left[\zeta_{\alpha}, \zeta_{\beta}\right]\right)\left[Z / p^{n}\right]\right)^{t}\left[X, Y \mid X^{2}=Y^{2}=1\right] \\
& \oplus \prod_{\alpha\left|a_{p}^{\prime}, \beta\right| b}\left(\left(Z_{p} \otimes Z\left[\zeta_{\alpha}, \zeta_{\beta}\right]\right)\left[Z / p^{n}\right]\right)^{t}\left[X, Y \mid X^{2}=Y^{2}=-1\right] .
\end{aligned}
$$

We suppress everywhere the relations $X \zeta_{\alpha} X^{-1}=\zeta_{\alpha}^{-1}, Y \zeta_{\beta} Y^{-1}=\zeta_{\beta}^{-1}$. The top component involves only the blocks

$$
B_{\alpha, \beta,+}=\left(\left(Z_{p} \otimes Z\left[\zeta_{\alpha}, \zeta_{\beta}\right]\right)\left[Z / p^{n}\right]\right)^{t}\left[X, Y \mid X^{2}=Y^{2}=1\right]
$$

and

$$
B_{\alpha, \beta,-}=\left(\left(Z_{p} \otimes Z\left[\zeta_{\alpha}, \zeta_{\beta}\right]\right)\left[Z / p^{n}\right]\right)^{t}\left[X, Y \mid X^{2}=-1\right],
$$

where $(\alpha, \beta)$ is full with respect to $\left(a_{p}^{\prime}, b\right)$. We shall have to distinguish between the two cases: (1) when $P_{1}$ contains at least two prime powers, and (2) when $P_{1}$ contains only $p^{n}$. We first consider (1). We assume that $Q\left(\zeta_{\alpha \beta}\right) / Q\left(\eta_{\alpha}, \eta_{\beta}\right)$ does not split at $p$ (leaving the split case to the reader), so we assume that

$$
B_{\alpha, \beta,+}=\hat{Z}_{p}\left[\zeta_{\alpha}, \zeta_{\beta}\right]\left[Z / p^{n}\right]^{t}\left[X, Y \mid X^{2}=Y^{2}=1\right],
$$

and must calculate the $p^{\prime}$-part of

$$
\hat{D}\left(B_{\alpha, \beta,+}\right)=\frac{Z_{p}\left[\eta_{\alpha}, \eta_{\alpha}\right]^{\times} \oplus Z_{p}\left[\eta_{\alpha p}, \eta_{\beta}\right]^{\times} \oplus \ldots \oplus Z_{p}\left[\eta_{\alpha p^{n}, \eta_{\beta}}\right]^{\times}}{\operatorname{Nrd}\left(\left(B_{\alpha, \beta,+}\right)^{\times}\right)}
$$

Let $J$ be the ideal

$$
J=\left(A_{p}-1\right) B_{\alpha, \beta,+} \cdot
$$

This ideal is topologically nilpotent. Therefore we get an exact sequence 


$$
\left.0 \rightarrow(1+J)^{\times} \rightarrow B_{\alpha, \beta,+}^{\times} \rightarrow Z_{p}\left[\zeta_{\alpha}, \zeta_{\beta}\right]^{t}[X, Y] X^{2}=Y^{2}=1\right]^{\times} \rightarrow 0 .
$$

By a result of Auslander-Goldman-Rim, cf. [7, Theorem 40.14] the rightmost term is a maximal order, so by taking reduced norms we get the diagram

$$
\begin{aligned}
& 0 \longrightarrow \underset{\substack{(1+J)^{\times} \\
\downarrow N r d}}{(\longrightarrow, d,} \longrightarrow \mathrm{Z}_{p}\left[\zeta_{\alpha}, \zeta_{\beta}\right]^{t}\left[X, Y \mid X^{2}=Y^{2}=1\right]^{\times} \rightarrow 0
\end{aligned}
$$

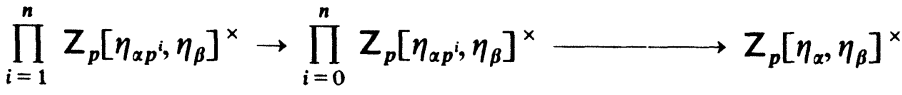

$$
\begin{aligned}
& \stackrel{\downarrow}{C_{J} \longrightarrow \hat{D}_{p}\left(B_{\alpha, \beta,+}\right) \longrightarrow} \longrightarrow
\end{aligned}
$$

If we let $N_{0}, N_{1}, \ldots, N_{n}$ denote the components of the reduced norm map on

$$
\mathrm{Q}_{p}\left(\zeta_{\alpha}, \zeta_{\beta}\right)\left[Z / p^{n}\right]^{t}\left[X, Y \mid X^{2}=Y^{2}=1\right]=\prod_{i=0}^{n} M_{4}\left(\mathrm{Q}_{p}\left(\eta_{\alpha p^{i}}, \eta_{\beta}\right)\right),
$$

then for $x \in B_{\alpha, \beta,+}$ we have the relations

$$
N_{i}(x) \equiv N_{j}(x) \quad \text { in } \quad F_{p} \otimes Z\left[\eta_{\alpha}, \eta_{\beta}\right], \quad \text { all } i, j .
$$

This is because $\zeta_{p^{i}}$ maps to 1 by reduction modulo primes above $p$. This relation implies that

$$
\operatorname{Nrd}\left((1+J)^{\times}\right) \subseteq \prod_{i=1}^{n} U_{1}\left(Z_{p}\left[\eta_{p_{\alpha}^{i}}, \eta_{\beta}\right]\right)
$$

where $U_{1}$ denotes the first unit group. Since $\left.\operatorname{Nrd}(1+J)^{\times}\right)$is open, there exist integers $m_{1}, \ldots, m_{n}$ such that

$$
\left.\prod_{i=1}^{n} U_{m_{i}}\left(Z_{p}\left[\eta_{\alpha p_{i}}, \eta_{\beta}\right]\right) \leqq N r d(1+J)^{\times}\right) .
$$

Hence the $p^{\prime}$-part of $C_{J}$ must be equal to

$$
\prod_{i=1}^{n}\left(F_{p} \otimes Z\left[\eta_{\alpha}, \eta_{\beta}\right]\right)^{\times}
$$

through reduction modulo the prime in $Z_{p}\left[\eta_{\alpha p}, \eta_{\beta}\right]$. Furthermore, the above relation implies that on the $p^{\prime}$-part, the map $C_{J} \rightarrow \hat{D}_{P}\left(B_{\alpha, \beta,+}\right)$ is injective and hence

$$
\hat{D}_{p}\left(B_{\alpha, \beta,+}\right)_{\left(p^{\prime}\right)} \cong \prod_{i=1}^{n}\left(F_{p} \otimes Z\left[\eta_{\alpha}, \eta_{\beta}\right]\right)^{\times}
$$

via the map

$$
\left(x_{0}, x_{1}, \ldots, x_{n}\right) \rightarrow \prod_{i=1}^{n}\left(\varrho_{p}\left(x_{i} x_{0}^{-1}\right)\right),
$$


where $\varrho_{p}$ means reduction modulo the appropriate prime over $p$.

If $P_{1}$ contains only $p^{n}$, then the group algebra splits in a little different way:

$$
\begin{aligned}
\mathrm{Q}\left(\zeta_{\beta}\right)\left[\mathrm{Z} / p^{n}\right]^{t} & {\left[X, Y \mid X^{2}=Y^{2}=1\right] } \\
& =M_{2}\left(\mathrm{Q}\left(\eta_{\beta}\right)\right)_{x=1} \oplus M_{2}\left(\mathrm{Q}\left(\eta_{\beta}\right)\right)_{x=-1} \oplus \prod_{i=1}^{n} M_{4}\left(\mathrm{Q}\left(\eta_{p^{i}}, \eta_{\beta}\right)\right) .
\end{aligned}
$$

If we let $N_{0,1}, N_{0,-1}, N_{1}, \ldots, N_{n}$ denote the components of the reduced norm corresponding to this decomposition, we get the relations

$$
N_{0,1}(x) N_{0,-1}(x) \equiv N_{i}(x) \quad \text { in }\left(F_{p} \otimes Z\left[\eta_{\beta}\right]\right)
$$

for $x \in Z_{p}\left[\zeta_{\beta}\right]\left[Z / p^{n}\right]^{t}\left[X, Y \mid X^{2}=Y^{2}=1\right], i>0$. This is essentially contained in section of [1]. Now we can argue like in the previous case and find that the $p^{\prime}-$ part of $\hat{D}_{p}\left(B_{\alpha, \beta,+}\right)$ is equal to $\prod_{i=1}^{n}\left(F_{p} \otimes Z\left[\eta_{\alpha}, \eta_{\beta}\right]\right)^{\times}$via the map

$$
\left(x_{0,1}, x_{0,-1}, x_{1}, \ldots, x_{n}\right) \rightarrow \prod\left(\varrho_{p}\left(x_{i} x_{0,1}^{-1} x_{0,-1}^{-1}\right)\right) .
$$

When using the same technique to calculate $\hat{D}_{p}\left(B_{\alpha, \beta,-}\right)$ we get the twisted group ring $Z_{p}\left[\zeta_{\alpha}, \zeta_{\beta}\right]^{t}\left[X, Y \mid X^{2}=Y^{2}=-1\right]$, where $P_{1}$ contains at least two prime powers, and we must prove that the reduced norm is surjective on the units of this ring. But $Q_{p}\left(\zeta_{\alpha}, \zeta_{\beta}\right) / Q\left(\eta_{\alpha}, \eta_{\beta}\right)$ is unramified, so the ring is a hereditary order by a result of Williamson-Harada, cf. [7, Theorem 40.15]. From the structure theorem for local hereditary orders, [7, Theorem 39.14], it is obvious that the reduced norm is surjective. One could also argue that since $\operatorname{Trd}(X)=\operatorname{Trd}(Y)=\operatorname{Trd}(X Y)=0$, the proof of [7, Theorem 40.14] carries over to show that the above group ring is actually a maximal order. In any case, the above method applies and we find that the $p^{\prime}$-part of $\hat{D}_{p}\left(B_{\alpha, \beta,-}\right)$ is equal to $\prod_{i=1}^{n}\left(F_{p} \otimes Z\left[\eta_{\alpha}, \eta_{\beta}\right]\right)^{\times}$if $P_{1}$ contains at least two prime powers. If $P_{1}$ contains only $p^{n}$, the calculation is a little more complicated. The group algebra splits like

$$
\mathrm{Q}_{p}\left(\zeta_{4 \beta}\right)^{t}\left[Y \mid Y^{2}=-1\right] \oplus \prod_{i=1}^{n} \mathrm{Q}_{p}\left(\zeta_{p}, \zeta_{\beta}\right)^{t}\left[X, Y \mid X^{2}=Y^{2}=-1\right]
$$

Letting $N_{0}, N_{1}, \ldots, N_{n}$ denote the components of the reduced norm, we get the relation

$$
\left(N \circ N_{0}\right)(x) \equiv N_{i}(x), \quad i>0,
$$

for $x \in Z_{p}\left[\zeta_{\beta}\right]\left[Z / p^{n}\right]^{t}\left[X, Y \mid X^{2}=Y^{2}=-1\right]^{\times}$. Here $N$ is the norm from $Q\left(\eta_{4 \beta}\right)$ to $\mathrm{Q}\left(\eta_{\beta}\right) \cong \mathrm{Q}\left(\eta_{p}, \eta_{\beta}\right)$. This is essentially contained in section 2 of [1]. Therefore, also in this case the $p^{\prime}$-part of $\hat{D}_{p}\left(B_{\alpha, \beta,-}\right)$ is equal to $\prod_{i=1}^{n}\left(F_{p} \otimes Z\left[\eta_{\beta}\right]\right)^{\times}$via the map

$$
\left(x_{0}, x_{1}, \ldots, x_{n}\right) \rightarrow \prod_{i=1}^{n}\left(\varrho_{p}\left(x_{i} N\left(x_{0}\right)^{-1}\right)\right)
$$


Finally, it is easy to see that $\tilde{E}_{P}\left(D_{2}(\pi)\right)_{(2)}$ is trivial. This proves Proposition 2.3.

We turn to the cyclic group $C$. If $p \| C \mid, p \neq 2$, we decompose

$$
\mathrm{Z}_{p} C=\prod_{\substack{\alpha\left|a_{p}^{\prime} \\ \beta\right| b}}\left(\left(\mathrm{Z}_{p} \otimes \mathrm{Z}\left[\zeta_{\alpha}, \zeta_{\beta}\right]\right)\left[\mathrm{Z} / p^{n}\right]_{T=1} \oplus\left(\mathrm{Z}_{p} \otimes \mathrm{Z}\left[\zeta_{\alpha}, \zeta_{\beta}\right]\right)\left[\mathrm{Z} / \dot{p}^{n}\right]_{T=-1}\right) .
$$

The top component involves only the blocks

$$
\begin{aligned}
& A_{\alpha, \beta,+}=\left(Z_{p} \otimes Z\left[\zeta_{\alpha}, \zeta_{\beta}\right]\right)\left[Z / p^{n}\right]_{T=1} \\
& A_{\alpha, \beta,-}=\left(Z_{p} \otimes Z\left[\zeta_{\alpha}, \zeta_{\beta}\right]\right)\left[Z / p^{n}\right]_{T=-1},
\end{aligned}
$$

where $(\alpha, \beta)$ is full with respect to $\left(a_{p}^{\prime}, b\right)$. By the same method as above we find that

$$
\tilde{E}_{P}\left(\hat{D}_{p}\left(A_{\alpha, \beta,}\right)\right)_{\left(p^{\prime}\right)} \cong \prod_{i=1}^{n}\left(F_{p} \otimes Z\left[\zeta_{\alpha}, \zeta_{\beta}\right]\right)^{\times}
$$

via reduction modulo primes above $p$. Furthermore it is easy to see that $\tilde{E}_{P}\left(\hat{D}_{2}\left(A_{\alpha, \beta, \pm}\right)\right)_{(2)}=0$.

According to the remark after Lemma 1.1 the maps $\operatorname{Res}_{C}^{\pi}$ and $\tilde{E}_{P}$ commute on the character rings. Hence $\operatorname{Res}_{C}\left(\widetilde{E}_{P}(\hat{D}(\pi))\right) \cong \widetilde{E}_{P}(\hat{D}(C))$. Comparing the results just obtained on the structure of $\tilde{E}_{P}(\hat{D}(\pi))$ and $\widetilde{E}_{P}(\hat{D}(C))$ with the tabulation of the induction map $R(C) \rightarrow R(\pi)$ (Table 2.5), we see that on $\tilde{E}_{P}(\hat{D}(\pi))_{(2)}$ the restriction map $\operatorname{Res}_{C}$ is the inclusion

$$
\prod_{(\alpha, \beta)} \prod_{i=1}^{n}\left(F_{p} \otimes Z\left[\eta_{\alpha}, \eta_{\beta}\right)_{(2)}^{\times} \rightarrow \prod_{(\alpha, \beta)} \prod_{i=1}^{n}\left(F_{p} \otimes Z\left[\zeta_{\alpha}, \zeta_{\beta}\right]\right)_{(2)}^{\times}\right.
$$

and therefore injective. Since $\tilde{E}_{P}\left(\hat{D}_{2}(\pi)\right)_{(2)}=0$, this completes the proof of Proposition 2.2.

\section{Proof of the main theorem.}

Lemma 3.1. $\sigma_{4}\left(e_{0}\right)$ is of order at most 2. If $S \cap P_{1}$ or $S \cap P_{2}$ is empty, then $\tilde{E}_{S}\left(\sigma_{4}\left(e_{0}\right)\right)=0$.

Proof. Let $\chi_{C}$ be the character on the cyclic group $C$ that restricts to $\chi_{p}$ on the $p$-part $(p \mid a b)$ and to the non-trivial character on $Z / 2$. Let $\psi_{C}=\operatorname{Ind}_{C}^{\pi}\left(\chi_{C}\right)$. By the Mackey decomposition theorem $\psi_{C}$ restricts to $2 \cdot\left(\chi_{p}+\chi_{p}^{-1}\right)$ on the $p$-part of $\pi$, for $p \mid a b$, and to $2 \cdot \chi_{2}$ on $\mathrm{Q}(8)$. In other words, $c_{4}\left(\psi_{C}\right)$ restricts like the cup product square $e_{0}^{2} \in H^{8}(\pi, \mathrm{Z})$ so $e_{0}^{2}=c_{4}\left(\psi_{C}\right)$. Hence $0=\sigma_{8}\left(e_{0}^{2}\right)=2 \cdot \sigma_{4}\left(e_{0}\right)$. This proves half of the lemma. To prove the second half it suffices to prove that $\tilde{E}_{S}\left(\sigma_{4}\left(e_{0}\right)\right)=0$ when $S=P_{1}$. By the naturality of $\sigma_{4}$ with respect to restriction 
and Proposition 2.1 it suffices to prove that $\sigma_{4}\left(\operatorname{Res}_{\pi\left(P_{1}\right)}^{\pi}\left(e_{0}\right)\right)=0$. Let $e$ $=\operatorname{Res}_{\pi\left(P_{1}\right)}^{\pi}\left(e_{0}\right)$. Then $e$ maps to $c_{2}\left(\chi_{p}+\chi_{p}^{-1}\right)$ by restriction to the $p$-part $(p \mid a)$ and to $c_{2}\left(\chi_{2}\right)$ by restriction to $Q(8)$. The group $Q(8 a, 1)$ contains the cyclic subgroup $C_{1}$ of order $4 a$. Let $\chi$ be the character on $C_{1}$ that restricts to $\chi_{p}$ (for $p \mid a$ ) on the $p$-part and to a faithful character on $Z / 4$. Let $\psi$ be the representation of $Q(8 a, 1)$ induced from $\chi$. Then $\psi$ restricts to $\chi_{p}+\chi_{p}^{-1}$ on the $p$ part $(p \mid a)$ and to $\chi_{2}$ on $Q(8)$. Hence $c_{2}(\psi)=e$ and therefore $\sigma_{4}(e)=0$.

Now we turn to the top component of $\sigma_{4}\left(e_{0}\right)$. In the previous section we found that

$$
\tilde{E}_{P}(\hat{D}(\pi))_{(2)}=\prod_{p \mid a b}\left(D_{p,+} \oplus D_{p,-}\right) .
$$

Let $\Sigma$ be the element of $\tilde{E}_{P}(\hat{D}(\pi))_{(2)}$ that decomposes as $\prod_{p \mid a}(1,4) \times$ $\prod_{p \mid b}(-1,4)$ where we consider $\boldsymbol{F}_{p}^{\times}$mapped diagonally into $\left(\boldsymbol{F}_{p} \otimes \mathbf{Z}\left[\eta_{\alpha}, \eta_{\beta}\right]\right)_{(2)}^{\times}$.

Proposition 3.2. The top component $\tilde{E}_{P}\left(\sigma_{4}\left(e_{0}\right)\right)$ is contained in the kernel group $D(\pi)$ and under the map $\partial: \tilde{E}_{P}(\hat{D}(\pi))_{(2)} \rightarrow \widetilde{E}_{P}(D(\pi))$ it is the image of $\Sigma$.

Proof. Corresponding to $e_{0}$ there is a periodic projective resolution of $\mathrm{Z}$ over $\mathrm{Z} \pi$. Following [8], this resolution defines an idele

$$
\hat{\tau}_{4}\left(e_{0}\right) \in J(Z(\mathrm{Q} \pi))_{+} / N r d(U(\mathrm{Z} \pi))
$$

and $\partial \hat{\tau}_{4}\left(e_{0}\right)=\sigma_{4}\left(e_{0}\right)$, cf. [8, Theorem 9.1]. Our calculations center around the diagram

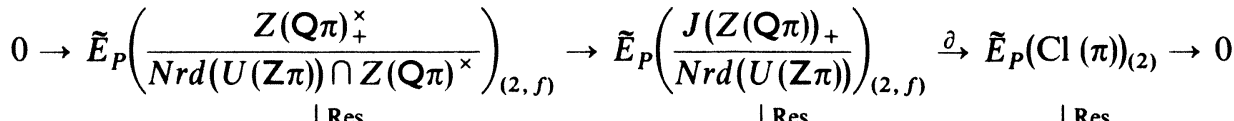

$$
\begin{aligned}
& 0 \longrightarrow \tilde{E}_{P}\left(\frac{\mathrm{Q} C^{\times}}{\operatorname{Nrd}(U(\mathrm{ZC})) \cap \mathrm{Q} C^{\times}}\right) \longrightarrow \tilde{E}_{P}\left(\frac{J(\mathrm{Q} C)}{\operatorname{Nrd}(U(\mathrm{ZC}))}\right) \stackrel{\partial}{\longrightarrow} \tilde{E}_{P}(\mathrm{Cl}(C)) \longrightarrow 0
\end{aligned}
$$

remembering that Res on $\tilde{E}_{P}\left(J(Z(\mathrm{Q} \pi))_{+} / N r d(U(\mathrm{Z} \pi))\right)_{(2, f)}$ is just inclusion of ideal groups and residue fields. When restricting to $C$ we have $\operatorname{Res}\left(e_{0}\right)=$ $c_{2}\left(\chi_{C}+\chi_{C}^{-1}\right)$, where $\chi_{C}$ is the faithful character on $C$ from Lemma 3.1. Hence $\operatorname{Res}\left(\tilde{E}_{P}\left(\sigma_{4}\left(e_{0}\right)\right)\right)=0$ and

$$
\tilde{E}_{P}\left(\hat{\tau}_{4}\left(\operatorname{Res}\left(e_{0}\right)\right)\right)=\tilde{E}_{P}\left(\tau_{4}\left(\operatorname{Res}\left(e_{0}\right)\right)\right),
$$

where $\tau_{4}$ is the Reidemeister torsion:

$$
\tau_{4}\left(\operatorname{Res}\left(e_{0}\right)\right) \in \mathrm{Q} C^{\times} / \mathrm{Q} C^{\times} \cap N r d(U(Z C)) .
$$


According to [6], $\tau_{4}\left(c_{2}\left(\chi_{C}+\chi_{C}^{-1}\right)\right)$ is the class of

$$
\begin{aligned}
& w_{\alpha, \beta, \pm 1} \rightarrow 2 \mp \eta_{\alpha \beta} \quad(\alpha|a, \beta| b ; \alpha \cdot \beta \neq 1) \\
& w_{1,1,1} \rightarrow\left(4 a^{2} b^{2}\right)^{-1} \\
& w_{1,1,-1} \rightarrow 4
\end{aligned}
$$

in $\mathrm{Q} C^{\times} / \mathrm{Q} C^{\times} \cap U(\mathrm{ZC})$. The action of $\tilde{E}_{P}$ on $J(\mathrm{Q} C)$ is easily calculated by restriction of characters, and we get:

If $(\alpha, \beta)$ is not full with respect to $(a, b)$, then $\tilde{E}_{P} w_{\alpha, \beta, \pm 1}=0$, while

$$
\begin{aligned}
\tilde{E}_{P} w_{\alpha, \beta, 1} & =w_{\alpha, \beta, 1}-W_{(1)}^{+}+W_{(2)}^{+}-\ldots+(-1)^{i} W_{(i)}^{+}+\ldots \\
\tilde{E}_{P} w_{\alpha, \beta,-1} & =w_{\alpha, \beta,-1}-W_{(1)}^{-}+W_{(2)}^{-}-\ldots+(-1)^{i} W_{(i)}^{-}+\ldots,
\end{aligned}
$$

where $W_{(i)}^{ \pm}$is the sum of all $w_{\gamma, \delta, \pm 1}$ with $\gamma \delta$ containing all prime powers in $\alpha \beta$ except $i$ of them.

Hence $\tilde{E}_{P}\left(\hat{\tau}_{4}\left(\operatorname{Res} e_{0}\right)\right)$ is the class of

$$
w_{\alpha, \beta, \pm 1} \rightarrow\left(2 \mp \eta_{\alpha \beta}\right) \bar{W}_{(1)}^{ \pm}(\alpha, \beta)^{-1} \bar{W}_{(2)}^{ \pm}(\alpha, \beta) \ldots \bar{W}_{(\bar{i})}^{ \pm}(\alpha, \beta)^{(-1)^{\prime}} \ldots
$$

in $J(\mathrm{Q} C) / U(\mathrm{ZC})$. Here $\bar{W}_{(i)}^{ \pm}(\alpha, \beta)$ is (for $\left.i<n\right)$ the product of all $2 \mp \eta_{\gamma \delta}$ with $\gamma \delta$ containing all prime powers in $\alpha \beta$ except $i$ of them and $\bar{W}_{(n)}^{+}(\alpha, \beta)=1 / 4 a^{2} b^{2}$ and $\bar{W}_{(n)}^{-}(\alpha, \beta)=4(n=$ card $(P))$. A typical factor in $\bar{W}_{(n-1)}^{ \pm}(\alpha, \beta)$ looks like $\left(2 \mp \eta_{p^{m}}\right)$, and since $\tilde{E}_{P}\left(Z(\mathrm{Q} \pi)_{+}^{\times}\right)$contains $\mathrm{Q}\left(\eta_{\alpha}, \eta_{\beta}\right)^{*} \supseteqq \mathrm{Q}\left(\eta_{p^{m}}\right)^{*}$, we see that $\tilde{E}_{P}\left(\sigma_{4}\left(e_{0}\right)\right)$ is the image under $\partial$ of the element $\xi$ of $\tilde{E}_{P}\left(J(Z(\mathrm{Q} \pi))_{+} / \operatorname{Nrd}(U(Z \pi))\right)$ that restricts to the element given by

$$
w_{\alpha, \beta, \pm 1} \rightarrow\left(2 \mp \eta_{\alpha \beta}\right) \bar{W}_{(\overline{1})}^{ \pm}(\alpha, \beta)^{-1} \ldots \bar{W}_{(n-2)}^{ \pm}(\alpha, \beta)^{(-1)^{n-2}}=X_{\alpha, \beta}^{ \pm} .
$$

Obviously $X_{\alpha, \beta}^{ \pm}$is a unit, so $\xi \in \hat{D}(\pi)$, and therefore $\tilde{E}_{P}\left(\sigma_{4}\left(e_{0}\right) \in D(\pi)\right.$. Since $\zeta_{p^{m}}$ maps to 1 by reduction module (primes above) $p$, we find that by reduction modulo $p$, most terms in $X_{\alpha, \beta}^{ \pm}$cancel out, and we are left with

$$
Y_{\alpha, \beta}^{+}=\left(\prod_{\substack{q^{\prime}|| \alpha \beta \\ q \neq p}}\left(2-\eta_{q^{j}}\right)\right)^{ \pm 1} \in\left(\boldsymbol{F}_{p} \otimes Z\left[\eta_{\alpha_{p}}, \eta_{\beta}\right]\right)^{\times}
$$

and

$$
Y_{\alpha, \beta}^{-}=\left(\prod_{\substack{q^{\prime}|| \alpha \beta \\ q \neq p}}\left(2+\eta_{q^{j}}\right)\right)^{ \pm 1} \in\left(\boldsymbol{F}_{p} \otimes Z\left[\eta_{\alpha_{p}^{\prime}}, \eta_{\beta}\right]\right)^{\times} .
$$

Here $\|$ means "exactly dividing". Adjusting $X_{\alpha, \beta}^{-}$by the number $\prod_{q^{\prime} \| \alpha \beta}(2$ $\left.+\eta_{q^{j}}\right) \in \mathrm{Z}\left[\eta_{\alpha}, \eta_{\beta}\right]^{*}$, we find that the $D_{p,-}$-components of $\tilde{E}_{P}\left(\sigma_{4}\left(e_{0}\right)\right)$ are exactly the $D_{p,-}$-components of $\Sigma$, namely 4 . To get the $D_{p,+}$-components we need the following lemma, guaranteeing the existence of a certain unit: 
Lemma 3.3. Let $S_{1}$ and $S_{2}$ be two sets of (relatively prime) prime powers. For each set $\mathscr{M}$ of prime powers, define

$$
\eta_{\mathscr{M}}= \begin{cases}2 & \text { if } \mathscr{M}=\varnothing \\ \eta_{x} & \text { if } x=\Pi p^{i}, \text { where } p^{i} \text { runs through } \mathscr{M}, \mathscr{M} \neq \varnothing .\end{cases}
$$

Let

$$
\begin{aligned}
t_{S_{1}, S_{2}, 2 i} & = \begin{cases}\prod_{\substack{S \subseteq S_{1} \cup S_{2} \\
\operatorname{card}(S)=2 i}}\left(\eta_{S \cap S_{1}}-\eta_{S \cap S_{2}}\right) & \text { if } 2 \leqq 2 i \leqq \operatorname{card}\left(S_{1} \cup S_{2}\right) \\
1 & \text { otherwise }\end{cases} \\
n_{S_{1}, S_{2}, 2 i+1} & = \begin{cases}\prod_{\substack{S \subseteq S_{1} \cup S_{2} \\
\operatorname{card}(S)=2 i+1}}\left(\left(\eta_{S \cap S_{1}}-\eta_{S \cap S_{2}}\right)\right. & \text { if } 3 \leqq 2 i+1 \leqq \operatorname{card}\left(S_{1} \cup S_{2}\right) \\
1 & \text { otherwise. }\end{cases}
\end{aligned}
$$

Put

$$
\begin{aligned}
& t_{S_{1}, S_{2}}=\prod_{i=1}^{\infty} t_{S_{1}, S_{2}, 2 i} \\
& n_{S_{1}, S_{2}}=\prod_{i=1}^{\infty} n_{S_{1}, S_{2}, 2 i+1} .
\end{aligned}
$$

Then $t_{S_{1}, S_{2}}$ and $n_{S_{1}, S_{2}}$ are units of $Z\left[\eta_{S_{1}}, \eta_{S_{2}}\right]$ and

$$
\varrho_{h}\left(\frac{t_{S_{1}, S_{2}}}{n_{S_{1}, S_{2}}}\right)= \begin{cases}(-1)^{\operatorname{card}\left(S_{1}\right)-1} \prod_{\substack{q^{i} \in S_{1} \cup S_{2} \\ q \neq p}}\left(2-\eta_{q^{i}}\right) & \text { if } p \in S_{1} \\ (-1)^{\operatorname{card}\left(S_{1}\right)} \prod_{\substack{q^{\prime} \in S_{1} \cup S_{2} \\ q \neq p}}\left(2-\eta_{q^{i}}\right) & \text { if } p \in S_{2} .\end{cases}
$$

Here $\not$ is any prime of $Z\left[\eta_{S_{1}}, \eta_{S_{2}}\right]$ lying above $p$.

The proof of this lemma goes by induction on card $\left(S_{1} \cup S_{2}\right)$ and is left to the reader. Applying this lemma to adjust $X_{\alpha^{\prime}, \beta^{\prime}}^{+}$we get $D_{p,+}$-components exactly as stated in Proposition 3.

Now we can easily prove the main theorem: The choice of almost linear generator is natural with respect to restriction to the subgroups $\pi(S)$. By Corollary 1.2, Proposition 2.1, and Proposition 3.2 we can therefore conclude that $\tilde{E}_{S}\left(\sigma_{4}\left(e_{0}\right)\right)$ is zero for all $S$ with $S \cap P_{1} \neq \varnothing, S \cap P_{2} \neq \varnothing$ exactly when the conditions (1) and (2) of the theorem are fulfilled. By Lemma 3.1 the other components of $\sigma_{4}\left(e_{0}\right)$ are always zero. This completes the proof of the main theorem. 


\section{REFERENCES}

1. S. Bentzen and Ib Madsen, On the Swan subgroup of certain periodic groups, Math. Ann. 264 (1983), 447-474.

2. A. Fröhlich, Locally free modules over arithmetic orders, J. Reine Angew. Math. 274/275 (1975), 112-124.

3. A. Fröhlich, Arithmetic and Galois module structure for tame extensions, J. Reine Angew. Math. 286/287 (1976), 380-440.

4. I. Hambleton and I. Madsen, Local surgery obstructions and space forms, Preprint, Aarhus University, 1984.

5. R. J. Milgram, Evaluating the Swan finiteness obstruction for periodic groups, Preprint, Stanford, 1979.

6. J. Milnor, Whitehead torsion, Bull. Amer. Math. Soc. 72 (1966), 358-426.

7. I. Reiner, Maximal orders (London Math. Soc. Monographs 5), Academic Press, London - New York - San Francisco, 1975.

8. C. T. C. Wall, Periodic projective resolutions, Proc. London Math. Soc. 39 (1979), 509-553.

MATEMATISK INSTITUT

AARHUS UNIVERSITET

NY MUNKEGADE 116

8000 AARHUS C

DENMARK 